\title{
Efecto de bacterias probióticas en el cultivo larvario del ostión de placer Crassostrea corteziensis (Bivalvia: Ostreidae)
}

\author{
Angel Isidro Campa-Córdova², Antonio Luna-González¹, José Manuel Mazón-Suastegui², \\ Gabriel Aguirre-Guzmán ${ }^{3}$, Felipe Ascencio ${ }^{2}$ \& Héctor Abelardo González-Ocampo ${ }^{1 *}$ \\ 1. Centro Interdisciplinario de Investigación para el Desarrollo Integral Regional, Unidad Sinaloa, Boulevard Juan de \\ Dios Bátiz Paredes 252, Col. San Joachin, C.P. 81101, Guasave, Sinaloa, México; hgocampo@yahoo.com, \\ aluna@ipn.mx \\ 2. Centro de Investigaciones Biológicas del Noroeste, S.C. (CIBNOR), Mar Bermejo 195, Colonia Playa Palo de Santa \\ Rita, C.P. 23090, La Paz, Baja California Sur, México; jmazon04@cibnor.mx, ascencio@cibnor.mx, \\ angcamp04@cibnor.mx \\ 3. Facultad de Medicina Veterinaria y Zootecnia, Universidad Autónoma de Tamaulipas, Km 5 Carretera Ciudad \\ Victoria-Mante, C.P. 87000, Tamaulipas, México; gabaguirre@uat.edu.mx \\ * Correspondencia.
}

Recibido 22-III-2010. Corregido 26-IX-2010. Aceptado 27-X-2010.

\begin{abstract}
Effect of probiotic bacteria on survival and growth of Cortez oyster larvae, Crassostrea corteziensis (Bivalvia: Ostreidae). Disease control problems have major constraints in aquaculture production, and the use of probiotics in larviculture is a valid alternative to antibiotics. This study analyzed the effect of probiotic bacteria on survival and final size of Cortez oyster larvae Crassostrea corteziensis. Two different probiotic concentrations were evaluated, $1 \times 10^{4}$ and $1 \times 10^{5} \mathrm{CFU} / \mathrm{ml}$ of Lactic acid bacteria (strain NS61) isolated from Nodipecten subnodosus, and bacilli isolated from the white leg shrimp, Litopenaeus vannamei (Pseudomonas aeruginosa, strain YC58) and C. corteziensis (Burkholderia cepacia, strain Y021). Bacteria were added directly into culture tanks, starting the bioassays from veliger to pediveliger stages as follows: (1) Control, without probiotics; (2) lactic acid bacteria (Lb); (3) bacilli mix (Mb) in a proportion 1:1. Results showed a higher larval survival with $\mathrm{Lb}$ and $\mathrm{Mb}$ at a dose of $1 \times 10^{4} \mathrm{CFU} / \mathrm{ml}$ compared to the control group. Larvae exposed to $\mathrm{Mb}$ at $1 \times 10^{5} \mathrm{CFU} / \mathrm{ml}$ showed higher survival than $\mathrm{Lb}$ and control. Larval final size was not significantly increased with the tested probiotics, but larvae treated with $\mathrm{Lb}$ at $1 \times 10^{5} \mathrm{CFU} / \mathrm{ml}$ showed less survival rate than those treated at $1 \times 10^{4} \mathrm{CFU} / \mathrm{ml}$. This study showed the beneficial effect of these probiotics, added individually or mixed in $C$. corteziensis larvae culture. Rev. Biol. Trop. 59 (1): 183-191. Epub 2011 March 01.
\end{abstract}

Key words: probiotic bacteria, larvae, Crassostrea corteziensis, survival, growth.

La acuicultura es una actividad importante ante la demanda creciente de productos acuícolas a nivel mundial (Brugère \& Ridler 2004). La acuicultura de moluscos bivalvos representa una alternativa económicamente viable debido a la posibilidad de operación a gran escala. Esta actividad puede ser ambientalmente sostenible al coadyuvar en la reducción del esfuerzo pesquero en la zona costera (Pipitone et al. 2000), además, los moluscos bivalvos pueden fungir como posibles biomonitores para cuantificar los niveles de contaminación marina (Gifford et al. 2004) y reducir la eutrofización, gracias a su capacidad filtradora que les permite obtener alimento del medio marino, que incluye microorganismos y material orgánico particulado (Hawkins et al. 2001, Lindhal et al. 2005, Mazón-Suástegui et al. 2009).

El ostión de placer u ostra del Cortés (Crassostrea corteziensis) habita en las costas del Pacífico desde Panamá hasta el Golfo de California y se considera como una especie 
con potencial para ser cultivada en gran escala. Sin embargo, al igual que en otros bivalvos, la alta mortalidad que se presenta durante la etapa larvaria y juvenil, es el principal problema que limita el desarrollo del cultivo en el laboratorio. Las mortalidades de $C$. corteziensis, son generalmente provocadas por bacterias del género Vibrio como ocurre también en Argopecten purpuratus (Riquelme et al. 1995), A. ventricosus, Nodipecten subnodosus y Atrina maura (Luna-González et al. 2002), C. virginica (Elston \& Leibovitz 1980, Gómez-León et al. 2008), C. gigas (Sugumar et al. 1998, Luna-González et al. 2002), Pecten maximus (Lambert et al. 1999), Ruditapes philippinarum (Borrego et al. 1996, Abasolo-Pacheco et al. 2009).

En los cultivos larvarios de moluscos bivalvos frecuentemente se utilizan antibióticos para prevenir la mortalidad de larvas y juveniles (Luna-González et al. 2004). Sin embargo, existe una preocupación extendida acerca del uso de antibióticos, ya que éstos han permitido el surgimiento de bacterias resistentes (Inglis 1996). Un método alternativo al uso de antibióticos y que está ganando aceptación en la acuicultura es el uso de bacterias probióticas para controlar patógenos microbianos (Gómez-Gil et al. 2000, Robertson et al. 2000, Balcázar et al. 2006). Los productos probióticos han sido exitosos en la prevención de enfermedades bacterianas en moluscos (Macey \& Coyne 2005), peces (Gram et al. 1999, Robertson et al. 2000) y crustáceos (Harzevili et al. 1998, Rengpipat et al. 2000). Entre los efectos benéficos se encuentran un mayor crecimiento, eficiencia en la alimentación y mejoramiento de la respuesta inmune (Venkat et al. 2004). Varios estudios con probióticos han mostrado un control sobre infecciones generadas por $V$. tubiashii en larvas de $C$. gigas (Gibson et al. 1998), la inhibición de Vibrio sp. en larvas de P. maximus (Ruiz-Ponte et al. 1999) y A. purpuratus (Riquelme et al. 2000), además de la mejora en la supervivencia, el crecimiento y la resistencia a enfermedades en Haliotis midae (Macey \& Coyne 2005).
En este trabajo se evaluó el efecto de microorganismos con potencial probiótico en el crecimiento y supervivencia de larvas de C. corteziensis, cultivadas en el laboratorio, como una medida que pueda ayudar a mejorar el desarrollo de estos bivalvos en beneficio del sector acuícola. Se determinó la capacidad antagónica in vitro de estos probióticos potenciales en contra de $V$. alginolyticus y V. parahaemolyticus, como una posible herramienta de control profiláctico.

\section{MATERIALES Y MÉTODOS}

Obtención de bacterias probióticas: Pseudomonas aeruginosa (cepa YC58) y $B$. cepacia (cepa Y021) fueron aisladas de adultos de camarón blanco (Litopenaeus vannamei) y de ostión de placer (Crassostrea corteziensis), respectivamente (Luis-Villaseñor 2007). La bacteria ácido láctica (BAL), cepa NS61, fue aislada del intestino de almeja mano de león, Nodipecten subnodosus (Nava-Hernández 2008). Los aislados fueron caracterizados mediante tinción Gram, morfología celular, actividad hemolítica (Koneman et al. 2001) y actividad antagónica (Apún-Molina et al. 2009) contra dos cepas bacterianas patógenas, Vibrio alginolyticus (APSA 2) obtenida de la colección del Centro de Investigaciones Biológicas del Noroeste, S.C. (CIBNOR) en La Paz, Baja California Sur, México y V. parahaemolyticus (CAIM 170) obtenida del Centro Investigación en Alimentación y Desarrollo. A. C. (CIAD), Mazatlán, Sinaloa, México.

Cultivo de microorganismos: La cepa NS61 fue cultivada en placas con el medio de Mann, Rogosa y Sharp (MRS agar, Difco, Detroit MI, USA; \# cat. 288130) con $3 \%$ de $\mathrm{NaCl}$ e incubadas a $30^{\circ} \mathrm{C}$ por $72 \mathrm{~h}$ en jarras de anaerobiosis con el sistema GASPAK (BBL), resembrada para cultivos masivos (agar MRS) y almacenada a $-80^{\circ} \mathrm{C}$ en microtubos con medio MRS caldo y $15 \%$ (v/v) de glicerol (Whitman \& MacNair 2004). Pseudomonas aeruginosa (cepa YC58) y Burkholderia cepacia (cepa Y021) fueron cultivadas en placas con medio 
agar YPD (Sigma, \#cat. Y-1500; St. Louis MO, USA) con $3 \%$ de $\mathrm{NaCl}$ e incubadas a $37^{\circ} \mathrm{C}$ por 24h (Buller 2004, Whitman \& MacNair 2004). Estas cepas fueron de nuevo cultivadas en medio caldo YPD (DIFCO laboratorios) con $3 \%$ de $\mathrm{NaCl}$, cosechadas por centrifugación ( 8 $000 \times \mathrm{g}$ ) y almacenadas para su uso posterior a $-80^{\circ} \mathrm{C}$ con $15 \%$ de glicerol.

Para el conteo celular, cada cultivo bacteriano se suspendió en $1 \mathrm{ml}$ de solución salina estéril $(\mathrm{NaCl} 3 \%)$. La solución bacteriana se ajustó a una densidad óptica de uno en un espectrofotómetro Thermo Spectronic Genesys 2 (Thermo Scientific, Rochester, USA) a una longitud de onda de $540 \mathrm{~nm}$ para obtener una concentración de $1 \times 10^{9} \mathrm{cél} / \mathrm{ml}$ (Harzevili et al. 1998). A partir de esa concentración, se hicieron diluciones para ajustar la concentración bacteriana requerida en los tanques de cultivo.

Obtención de larvas de $\boldsymbol{C}$. corteziensis: En el Laboratorio de Reproducción de Especies Marinas del CIBNOR, se establecieron cultivos larvarios stock como respaldo y materia prima para los experimentos programados, aplicando procedimientos descritos por Mazón-Suástegui et al. (2002 y 2009). Los reproductores se transportaron en seco desde Bahía de Ceuta, Sinaloa y se mantuvieron bajo condiciones controladas $\left(26 \pm 0.5^{\circ} \mathrm{C}\right.$ y $37 \pm 0.5$ ups $)$. El agua de mar para el cultivo de las microalgas fue previamente irradiada con luz UV y conducida a través de un filtro de cartucho de $1 \mu \mathrm{m}$ (Torkildsen \& Magnesen 2004). Los reproductores se alimentaron continuamente con una mezcla 1:1:2 de Isochrysis galbana, Chaetoceros calcitrans y Ch. muelleri (=gracilis) $\left(2.4 \times 10^{6}\right.$ células/ organismo/día). El desove fue inducido mediante choque térmico $\left(18-28^{\circ} \mathrm{C}\right)$ y los óvulos fueron fertilizados añadiendo esperma fresco muy activo, en proporción 4:1. Las larvas se mantuvieron en tanques de fibra de vidrio de $50001\left(24 \pm 1^{\circ} \mathrm{C}\right.$ y $37 \pm 0.5$ ups $)$, con agua de mar filtrada por arena, cartuchos de 5 y $1 \mu \mathrm{m}$ y carbón activado, se utilizó como alimento una mezcla 1:1 de I. galbana y Ch. calcitrans, a razón de 30 000cél/ml.
Evaluación de cepas probióticas en cultivos larvarios de $\boldsymbol{C}$. corteziensis: La cepa BAL NS61 y la mezcla de dos cepas de bacilos (YC58 y Y021, en proporción 1:1), ambas se utilizaron en dos diferentes concentraciones: $1 \times 10^{4}$ y $1 \times 10^{5} \mathrm{UFC} / \mathrm{ml}$ con base en los resultados obtenidos de los trabajos de Luis-Villaseñor (2007) y Nava-Hernández (2008). Los dos bioensayos, con una duración de nueve y 11 días respectivamente, se realizaron en el laboratorio de larvicultura del CIBNOR. Se colocaron larvas véliger (3 larvas $/ \mathrm{ml}$ ) de $C$. corteziensis de cinco días de edad en tanques cilíndricos de fibra de vidrio con 301 de agua de mar filtrada $(1 \mu \mathrm{m})$ a temperatura de $29 \pm 1^{\circ} \mathrm{C}$, salinidad de 36ups y aireación constante. Los tratamientos se realizaron por triplicado de la siguiente manera: (1) tratamiento control, sin bacterias probióticas; (2) tratamiento con bacterias ácido lácticas (Lb) y (3) tratamiento con la mezcla de bacilos $(\mathrm{Mb})$ proporción 1:1. El cultivo larvario se realizó con recambio de agua del $100 \%$ cada $48 \mathrm{~h}$, agregando las bacterias correspondientes a cada tratamiento después de cada recambio. Las larvas fueron alimentadas diariamente con una mezcla de I. galbana, Pavlova lutherii y $C$. gracilis (1:1:1) a una concentración de $3.5 \times 10^{4}$ cél $/ \mathrm{ml}$. La supervivencia larvaria se determinó con un microscopio de contraste de fases (10x), se registró el número de larvas con nado normal contra el número de larvas moribundas, se observaron las valvas cerradas y sin movimiento del velo. Se tomaron muestras de $100 \mathrm{ml}$ de cada tanque de cultivo para determinar talla de 30 larvas tomadas al azar en un microscopio de contraste de fases. El experimento tuvo una duración máxima de 11 días, al llegar las larvas a estadio pediveliger.

Para comparar diferencias en la supervivencia y la talla de larvas en función del probiótico suministrado se hizo un análisis de varianza de una vía (ANOVA). Los valores de $\mathrm{p}<0.05$ fueron considerados significativamente diferentes. Cuando existieron diferencias significativas, se utilizó un análisis a posteriori y la prueba de Tukey (HSD) para identificar la naturaleza de estas diferencias $(\mathrm{p}<0.05)$. 


\section{RESULTADOS}

Supervivencia larvaria de $C$. corteziensis: La Fig. 1 muestra la supervivencia larvaria de Crassostrea corteziensis cultivadas durante nueve días con Lb y una mezcla (1:1) de $\mathrm{Mb}$ a una concentración de $1 \times 10^{4} \mathrm{UFC} / \mathrm{ml}$. La supervivencia larvaria fue significativamente mayor en los tratamientos $\mathrm{Mb}$ (Tukey, $\mathrm{p}<0.05)$ y $\mathrm{Lb}$ (Tukey, $\mathrm{p}<0.05$ ) que las larvas del grupo control.

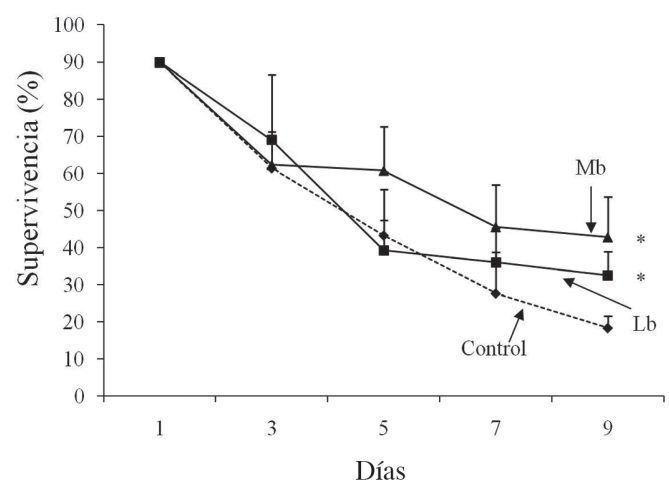

Fig. 1. Supervivencia larvaria de Crassostrea corteziensis cultivadas durante nueve días con lactobacilos (Lb) y una mezcla de bacilos (Mb) a una concentración de $1 \times 10^{4} \mathrm{UFC} /$ ml. Los datos son expresados como media \pm desviación estándar. *Significativamente diferente respecto al control $(\mathrm{p}<0.05)$.

Fig. 1. Larval survival of Crassostrea corteziensis cultured during nine days with lactobacilli (Lb), and a bacilli mix $(\mathrm{Mb})$ at $1 \times 10^{4} \mathrm{CFU} / \mathrm{ml}$. Data are expressed as mean \pm S.D. $*$ Significantly different than control $(\mathrm{p}<0.05)$.

La Fig. 2 se presenta la supervivencia larvaria de $C$. corteziensis cultivadas durante 11 días con $\mathrm{Lb}$ y $\mathrm{Mb}$ a una concentración de $1 \times 10^{5} \mathrm{UFC} / \mathrm{ml}$. Las larvas tratadas con Mb registraron una supervivencia significativamente mayor (Tukey, $\mathrm{p}<0.05$ ) que las larvas tratadas con Lb y que el grupo control. Los cultivos larvarios tratados con $\mathrm{Lb}$ disminuyeron su supervivencia al aumentar la concentración de la bacteria de $1 \times 10^{4} \mathrm{UFC} / \mathrm{ml}(32.5 \%)$ a $1 \times 10^{5} \mathrm{UFC} / \mathrm{ml}$ (14.7\%) (Figs. 1 y 2 ).

Talla final de $C$. corteziensis: La talla final larvaria de Crassostrea corteziensis

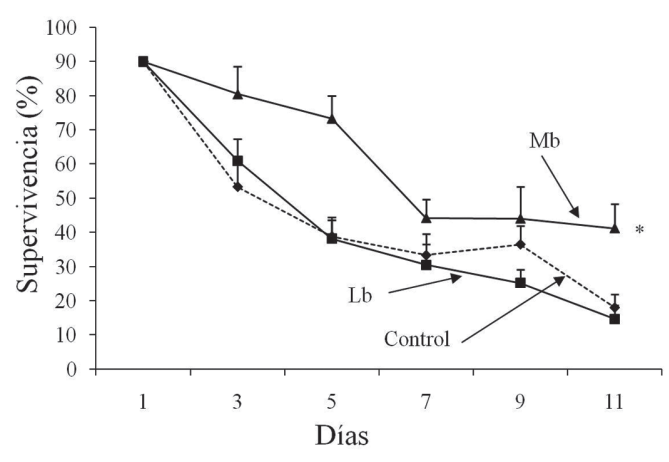

Fig. 2. Supervivencia larvaria de Crassostrea corteziensis cultivadas durante 11 días con lactobacilos (Lb) y una mezcla de bacilos (Mb) a una concentración de $1 \times 10^{5} \mathrm{UFC} /$ ml. Los datos son expresados como media \pm desviación estándar. *Significativamente diferente respecto al control $(\mathrm{p}<0.05)$.

Fig. 2. Larval survival of Crassostrea corteziensis cultured during 11 days with lactobacilli (Lb) and a bacilli mix $(\mathrm{Mb})$ at $1 \times 10^{5} \mathrm{CFU} / \mathrm{ml}$. Data are expressed as mean \pm S.D. *Significantly different than control $(\mathrm{p}<0.05)$.

obtenida fue la siguiente: en el tratamiento Control, la talla final en el experimento A fue de $169.54 \pm 25.5 \mu \mathrm{m}$ mientras que en el tratamiento B fue de $161.6 \pm 24.1 \mu \mathrm{m}$; en el tratamiento de lactobacilos (Lb) en el experimento A la talla final fue de $163.9 \pm 26.8 \mu \mathrm{m}$, mientras que en el tratamiento B fue de $142.8 \pm 27.1 \mu \mathrm{m}$ a una concentración de $1 \times 10^{4} \mathrm{UFC} / \mathrm{ml}$ en el primero y de $1 \times 10^{5} \mathrm{UFC} / \mathrm{ml}$ en el segundo; finalmente en el tratamiento de la mezcla de bacilos $(\mathrm{Mb})$ en el experimento A la talla final fue de $195.6 \pm 32.7 \mu \mathrm{m}$ mientras que en el experimento B fue de $188.7 \pm 29.2 \mu \mathrm{m}$.

La talla final de $C$. corteziensis cultivados con probióticos durante nueve días a una concentración de $1 \times 10^{4} \mathrm{UFC} / \mathrm{ml}$ no mostró diferencias significativas (Tukey, $\mathrm{p}>0.05$ ) entre las larvas tratadas con probióticos y las larvas del grupo control. Sin embargo, las larvas cultivadas con $\mathrm{Mb}$ registraron una talla promedio mayor $(189.5 \mu \mathrm{m})$ que las tratadas con $\mathrm{Lb}$ $(163.9 \mu \mathrm{m})$ y que las larvas control $(169.64 \mu \mathrm{m})$.

El crecimiento larvario de $C$. corteziensis cultivados con probióticos a una concentración de $1 \times 10^{5} \mathrm{UFC} / \mathrm{ml}$ no registró un incremento 
significativo (Tukey, $\mathrm{p}>0.05$ ) respecto al control. Se registró un incremento en el crecimiento promedio de las larvas tratadas con $\mathrm{Mb}(188.7 \mu \mathrm{m})$ respecto a las tratadas con $\mathrm{Lb}$ $(142.8 \mu \mathrm{m})$ y los controles $(161.6 \mu \mathrm{m})$. Los cultivos larvarios tratados con $\mathrm{Lb}$ disminuyeron su crecimiento al aumentar la concentración de $1 \times 10^{4} \mathrm{UFC} / \mathrm{ml}(163.9 \mu \mathrm{m})$ a $1 \times 10^{5} \mathrm{UFC} / \mathrm{ml}$ $(142.8 \mu \mathrm{m})$.

\section{DISCUSIÓN}

El cultivo larvario de moluscos bivalvos es una actividad en donde existen problemas debido a las mortalidades masivas que ocurren repentinamente, lo cual limita el éxito de los cultivos (Luna-González et al. 2004, Farzanfar 2006). Para prevenir estos eventos, frecuentemente se utilizan antibióticos de manera indiscriminada, lo que provoca resistencia de múltiples microorganismos patógenos en los estanques de cultivo (Vine et al. 2006, Castillo-Machalskis et al. 2007), por lo que el uso de probióticos representa una alternativa que puede sustituir el uso de aquellos (Balcázar et al. 2006) y genera a su vez un mejoramiento de la supervivencia y crecimiento de los organismos.

Para la selección de probióticos, es común realizar bioensayos de antagonismo in vitro, en donde patógenos son expuestos a las cepas candidatas a probióticos (Balcázar et al. 2006, Mantilla-Paredes et al. 2009). En el presente estudio, se seleccionaron tres cepas potencialmente probióticas por presentar actividad antagónica contra Vibrio harveyi y $V$. alginolyticus (Lactobacillus sp., cepa NS61; $P$. aeruginosa, cepa YC58 y B. cepacia, cepa Y021). La efectividad in vitro de un probiótico no garantiza los mismos resultados in vivo. Por ejemplo, Gram et al. (2001) reportaron que el antagonismo in vitro de Pseudomonas fluorescens (Cepa AH2) no mostró protección contra la furunculosis causada por Aeromonas salmonicida en cultivos de salmón del Atlántico, sin embargo resultó un efectivo probiótico contra vibriosis en el cultivo de trucha arcoiris.
Balcazar et al. (2006) recomiendan que los agentes probióticos deben ser aislados preferentemente del propio hospedero, no deben ser patogénicos y tener la capacidad de sobrevivir en el tracto intestinal del hospedero. El aislamiento de probióticos del intestino del propio organismo hospedero, se considera más apropiado debido a que estas bacterias benéficas están adaptadas a tolerar condiciones extremas en el tracto digestivo, además de la habilidad de adherirse a la superficie intestinal (Caipang et al. 2010). La habilidad de los probióticos para colonizar las células epiteliales de intestino, es un factor muy importante a considerar para prevenir la colonización de patógenos (Vine et al. 2004).

La mayoría de los probióticos propuestos en acuicultura pertenecen a los géneros Aeromonas sp., Bacillus sp., Carnobacterium sp., Lactobacillus sp., Flavobacterium sp., Pseudomonas sp., y Vibrio sp.. Bacterias Gramnegativas como Pseudomonas sp. y Vibrio sp. constituyen la microbiota nativa predominante en especies marinas (Otta et al. 1999). En el presente estudio, una mezcla de bacilos $(P$. aeruginosa, cepa YC58 y B. cepacia, cepa Y021, proporción 1:1) utilizada en concentraciones diarias de $1 \times 10^{4} \mathrm{UFC} / \mathrm{ml}$ y de $1 \times 10^{5} \mathrm{UFC} /$ $\mathrm{ml}$, incrementaron la supervivencia larvaria de C. corteziensis (Figs. 1 y 2). Las especies de Pseudomonas habitan comúnmente en suelo, agua dulce, agua marina en donde producen un amplio rango de metabolitos secundarios como antibióticos, cianuro de hidrógeno, sideróforos quelantes de hierro e inhiben un amplio intervalo de bacterias patógenas. Gram et al. (1999) observaron inhibición in vitro de $V$. anguillarum por $P$. fluorescens y una disminución en la mortalidad al utilizar el probiótico en el cultivo de peces de la especie Oncorhynchus mykiss. Además, Torrento \& Torres (1996) reportaron la inhibición in vitro de $V$. harveyi por $P$. aeruginosa.

Son pocos los estudios que reportan el uso de probióticos en el cultivo larvario de moluscos (Vine et al. 2006). Riquelme et al. (2000) reportaron el uso efectivo de Alteromona haloplanktis (cepa 77) y Vibrio sp. (cepa 
11) en el control de infecciones causadas por $V$. anguillarum en cultivos larvarios del pectínido Argopecten purpuratus. Ruiz-Ponte et al. (1999) mejoraron la supervivencia larvaria de Pecten maximus cuando se usa una mezcla de Roseobacter sp. (Cepa BS107) y V. anguillarum (cepa 408).

Se ha reportado el beneficio del uso de Lactobacillus sp. en salud humana debido a que reduce el colesterol, absorbe nutrientes, promueve la absorción de lactosa, modula la microbiota intestinal, previene el cáncer, infecciones virales y alergias (Kawahara \& Otani 2006). En este estudio, el uso de Lactobacillus sp. (cepa NS61) incrementó la supervivencia larvaria de $C$. corteziensis en una concentración de $1 \times 10^{4} \mathrm{UFC} / \mathrm{ml}$, similar al trabajo de Venkat et al. (2004) con larvas de Macrobrachium rosenbergii cultivadas con Lactobacillus sp. Sin embargo, el crecimiento larvario de $C$. corteziensis no fue significativo con los probióticos utilizados en este estudio. En este sentido, Campa-Córdova et al. (2009) reportaron un incremento en el crecimiento de juveniles de $C$. corteziensis mediante el uso de una dosis diaria de $5 \times 10^{4} \mathrm{UFC} / \mathrm{ml}$ de Lactobacillus sp. (Cepa NS61). Vine et al. (2006) recomiendan una dosis de probióticos en el agua de cultivo entre $1 \times 10^{4}$ y $1 \times 10^{6} \mathrm{UFC} / \mathrm{ml}$. Douillet \& Langdon (1994) incrementaron el crecimiento de C. gigas con una concentración de $1 \times 10^{5} \mathrm{UFC/}$ $\mathrm{ml}$ de la bacteria CA2. La exposición de $P$. fluorescens a juveniles de trucha arcoíris a una concentración de $1 \times 10^{5} \mathrm{UFC} / \mathrm{ml}$ redujeron la mortalidad a infecciones experimentales causadas por V. anguillarum. Peeters \& Rodríguez (1999) inocularon una bacteria probiótica a una concentración diaria de $1 \times 10^{5} \mathrm{UFC} / \mathrm{ml}$ en el cultivo larvario de camarón blanco Litopenaeus vannamei y previnieron la colonización de bacterias patógenas durante el cultivo larvario. Villamil-Díaz \& Martínez-Silva (2009) concluyen que los mayores beneficios obtenidos en acuicultura por el uso de probióticos son el incremento en la supervivencia durante infecciones experimentales, asociada a la potenciación de las defensas del sistema inmune.
Frecuentemente se utilizan probióticos sin un criterio científico, obteniendo en consecuencia resultados inconsistentes (Balcazar et al. 2006). El estudio de la interacción entre la microbiota intestinal y el hospedero es fundamental para el mejoramiento de los cultivos acuícolas. Los resultados de este estudio en el cultivo larvario de $C$. corteziensis muestran el beneficio de utilizar probióticos para mejorar la supervivencia. No obstante, se requieren estudios adicionales para evaluar diferente concentración/dosificación y determinar la que resulte más adecuada para mejorar la condición fisiológica de los organismos en cultivo y su respuesta inmune ante agentes patógenos y condiciones ambientales de estrés.

\section{AGRADECIMIENTOS}

A María de Jesús Romero por su apoyo técnico, así como por el apoyo económico al Centro de Investigaciones Biológicas del Noroeste a través del proyecto AC2.2, al proyecto SEP-CONACyT (25981) y al proyecto de la International Foundation of Science (IFS AA/14868R).

\section{RESUMEN}

El ostión de placer u ostra del Cortés (Crassostrea corteziensis) se considera como una especie con potencial para ser cultivada en gran escala. Sin embargo, al igual que en otros bivalvos, la alta mortalidad que se presenta durante la etapa larvaria y juvenil, es el principal problema que limita el desarrollo del cultivo en el laboratorio. Un método que está ganando aceptación en la acuicultura es el uso de bacterias probióticas para controlar patógenos microbianos. Este estudio analiza el efecto de estas bacterias en la supervivencia y talla final de larvas de ostión de placer Crassostrea corteziensis. Se utilizó una cepa de bacterias ácido lácticas (cepa NS61) aisladas N. subnodosus, así como de bacilos aislados de L. vannamei (Pseudomonas aeruginosa, cepa YC58) y de C. corteziensis (Burkholderia cepacia, cepa Y021). Las cepas se evaluaron por inmersión en cultivos larvarios de $C$. corteziensis a dos concentraciones diferentes, hasta completar el estadio pediveliger. Los organismos se trataron con bacterias ácido lácticas (Lb), una mezcla de bacilos (Lb) en proporción 1:1 y un grupo control. La concentración de $1 \times 10^{4} \mathrm{UFC} / \mathrm{ml}$ registró una mayor supervivencia con $\mathrm{Lb}$ y $\mathrm{Mb}$ respecto al grupo control. La supervivencia con $\mathrm{Mb}$ a una concentración 
de $1 \times 10^{5} \mathrm{UFC} / \mathrm{ml}$ fue mayor que la del grupo control y del grupo tratado con Lb. Los resultados mostraron que las larvas de C. corteziensis tratadas con probióticos no incrementaron significativamente su talla respecto a las larvas del grupo control. Mientras que las tratadas con Lb a la concentración mayor, $1 \times 10^{5} \mathrm{UFC} / \mathrm{ml}$, mostraron una disminución de la supervivencia respecto a las tratadas con $1 \times 10^{4} \mathrm{UFC} / \mathrm{ml}$. Este estudio demostró el efecto benéfico de cepas probióticas utilizadas individualmente o en mezcla en el cultivo larvario de $C$. corteziensis.

Palabras clave: Bacterias probióticas, larvas, Crassostrea cortezienzis, supervivencia, crecimiento.

\section{REFERENCIAS}

Abasolo-Pacheco, F., J.M. Mazón-Suástegui \& P.E. Saucedo. 2009. Response and condition of larvae of the scallops Nodipecten subnodosus and Argopecten ventricosus reared at the hatchery with different seawater sources. Aquaculture 296: 255-262.

Apún-Molina, J.P., A. Santamaría-Miranda, A. Luna-González, S.F. Martínez-Díaz \& M. Rojas-Contreras. 2009. Effect of potential probiotic bacteria on growth and survival of tilapia Oreochromis niloticus L., cultured in the laboratory under high density and suboptimum temperature. Aquac. Res. 40: 887-894.

Balcázar, J.L., I. de Blas, I. Ruiz-Zarzuela, D. Cunningham, D. Vendrell \& J.L. Múzquiz. 2006. The role of probiotics in aquaculture. Vet. Microbiol. 114: 173-186.

Borrego, J.J., D. Castro, A. Luque, C. Paillard, P. Maes, M.T. García \& A. Ventosa. 1996. Vibrio tapetis sp. nov. the causative agent of the brown ring disease affecting cultured clams. Int. J. Syst. Bacteriol. 46: 480-484.

Brugère, C. \& N. Ridler. 2004. Global aquaculture outlook in the next decades: an analysis of national aquaculture production forecasts to 2030. FAO Fisheries Circular No. 1001. FAO, Roma, Italia.

Buller, N.B. 2004. Bacteria from fish and other aquatic animals, a practical identification manual. CABI, Cambridge, Massachusetts, EEUU.

Caipang, C.M., M.F. Brinchmann \& V. Kiron. 2010. Antagonistic activity of bacterial isolates from intestinal microbiota of Atlantic cod, Gadus morhua, and an investigation of their inmmunomodulatory capabilities. Aquac. Res. 41: 249-256.

Campa-Córdova, A.I., H. González-Ocampo, A. LunaGonzález, J.M. Mazón-Suástegui \& F. Ascencio. 2009. Growth, survival, and superoxide dismutase activity in juvenile Crassostrea corteziensis (Hertlein, 1951) treated with probiotics. Hidrobiológica 19: 151-157.

Castillo-Machalskis, I., H. D'Armas, N. Malaver \& M. Núñez. 2007. Actividad antibacteriana de extractos de hongos aislados de raíces del mangle Rhizophora mangle (Rhizophoracea) en Venezuela. Rev. Biol. Trop. 55: 761-765.

Douillet, P.A. \& C.J. Langdon. 1994. Use of a probiotic for the culture of larvae of the Pacific oyster (Crassostrea gigas, Thunberg). Aquaculture 119: 25-40.

Elston, R.A. \& L. Leibovitz. 1980. Pathogenesis of experimental vibriosis in larval American oysters, Crassostrea virginica. Can. J. Fish Aquat. Sci. 37: 964-978.

Farzanfar, A. 2006. The use of probiotics in shrimp aquaculture. Federation of European Microbiological Societies. Immunol. Med. Microbiol. 48: 149-158.

Gibson, L.F., J. Woodworth \& A.M. George. 1998. Probiotic activity of Aeromonas media on the Pacific oyster, Crassostrea gigas, when challenged with Vibrio tubiashii. Aquaculture 169: 111-120.

Gifford, S., R.H. Dunstan, W. O'Connor, T. Roberts \& R. Toia. 2004. Pearl aquaculture - Profitable environmental remediation? Sci. Total Environ. 319: 27-37.

Gómez-Gil, B., A. Roque \& J.F. Turnbull. 2000. The use and selection of probiotic bacteria for use in the culture of larval aquatic organisms. Aquaculture 191: 259-270.

Gómez-León, J., L. Villamil, S.A. Salger, R.H. Sallum, A. Remacha-Triviño, D.F. Leavitt \& M. Gómez-Chiarri. 2008. Survival of eastern oysters Crassostrea virginica from three lines following experimental challenge with bacterial pathogens. Dis. Aquat. Organ. 79: 95-105.

Gram, L., J. Melchiorsen, B. Spanggaard, I. Huber \& T.F. Nielsen. 1999. Inhibition of Vibrio anguillarum by Pseudomonas fluorescens $\mathrm{AH} 2$, a possible probiotic treatment of fish. Appl. Environ. Microb. 65: 969-973.

Gram, L., T. Løvold, J. Nielsen, J. Melchiorsen \& B. Spanggaard. 2001. In vitro antagonism of the probiotic Pseudomonas fluorescens strain AH2 against Aeromonas salmonicida does not confer protection of salmon against furunculosis. Aquaculture 199: 1-11.

Harzevili, A.R.S., H. Van Duffel, P. Dhert, J. Swings \& P. Sorgeloos. 1998. Use of a potential probiotic Lactobacillus lactis Ar21 strain for the enhancement of 
growth in the rotifer Brachionus plicatillis (Müller). Aquac. Res. 29: 411-417.

Hawkins, A.J.S., J.G. Fang, P.L. Pascoe, J.H. Zhang, X.L. Zhang \& M.Y. Zhu. 2001. Modeling short-term responsive adjustments in particle clearance rate among bivalve suspension-feeders: separate unimodal effects of seston volume and composition in the scallop Chlamys farreri. J. Exp. Mar. Biol. Ecol. 262: 61-73.

Inglis, V. 1996. Antibacterial chemotherapy in aquaculture: review of practice, associated risks and need for action. Institute of Aquaculture, University of Stirling, Stirling, Escocia.

Kawahara, T. \& H. Otani. 2006. Stimulatory effect of lactic acid bacteria from commercial available nozawanazuke pickle on citokine expression by mouse spleen cells. Biosc. Biotechnol. Biochem. 70: 411-417.

Koneman, E.W., S.D. Allen, W.M. Janda, P.C. Schreckenberger \& W.C. Winn. 2001. Diagnóstico Microbiológico. Médica Panamericana, México D.F., México.

Lambert, C., J.L. Nicolas \& V. Cilia. 1999. Vibrio splendidus-related strain isolated from brown deposit in scallop (Pecten maximus) cultured in Brittany (France). Bull. Eur. Assoc. Fish Pathol. 19: 102-106.

Lindahl, O., R. Hart, B. Hernroth, S. Kollberg, L.O. Loo, L. Olrog, A.S. Rehnstam-Holm, J. Svensson, S. Svensson \& U. Syversen. 2005. Improving marine water quality by mussel farming: A profitable solution for Swedish society. Ambio 34: 131-138.

Luis-Villaseñor, I.E. 2007. Caracterización de microorganismos con potencial probiótico aislados del intestino del camarón blanco, Litopenaeus vannamei, y glándula digestiva de ostión de placer Crassostrea corteziensis. Tesis de Maestría, Universidad de Sonora, Hermosillo, México.

Luna-González, A., A.N. Maeda-Martinez, J.C. Sainz \& F. Ascencio-Valle. 2002. Comparative susceptibility of veliger larvae of four bivalve mollusks to a Vibrio alginolyticus strain. Dis. Aquat. Org. 49: 221-226.

Luna-González, A., A.N. Maeda-Martínez, F. AscencioValle \& M. Robles-Mungaray. 2004. Ontogenetic variations of hydrolytic enzymes in the Pacific oyster Crassostrea gigas. Fish Shell Immunol. 16: 287-294.

Macey, B.M. \& V.E. Coyne. 2005. Improved growth rate and disease resistance in farmed Haliotis midae through probiotic treatment. Aquaculture 245: 249-261.
Mantilla-Paredes, A.J., G.I. Cardona, C.P. Peña-Venegas, U. Murcia, M. Rodríguez \& M.M. Zambrano. 2009. Distribución de bacterias potencialmente fijadoras de nitrógeno y su relación con parámetros físicoquímicos en suelos con tres coberturas vegetales en el sur de la Amazonia colombiana. Rev. Biol. Trop. 57: 915-927.

Mazón-Suástegui, J.M., S. Avilés-Quevedo, M. RoblesMungaray \& F. Flores-Higuera. 2002. Experiencias en el cultivo de ostión de placer Crassostrea corteziensis a partir de semilla producida en el laboratorio. Memorias IV Simposium Nacional de Acuicultura y Pesca. Antigua, Guatemala.

Mazón-Suástegui, J.M., M.A. Parres-Haro, K.M. RuízRuíz, M.C. Rodríguez-Jaramillo \& P.E. Saucedo. 2009. Influence of hatchery diets on early grow-out of the Cortez oyster Crassostrea corteziensis in Guasave, Sinaloa, Mexico. Aquac. Res. 40: 1908-1914.

Nava-Hernández, J.C. 2008. Efecto de lactobacilos y bacilos con potencial probiótico, en el crecimiento y supervivencia de larvas y semillas de Nodipecten subnodosus (Sowerby, 1835) y Crassostrea corteziensis (Hertlein, 1951), cultivados en el laboratorio. Tesis de Maestría, Univesidad Autónoma de Baja California Sur, La Paz, México.

Otta, S.K., I. Karunasagar \& I. Karunasagar. 1999. Bacterial flora associated with shrimp culture ponds growing Penaeus monodon. J. Aquat. Trop. 14: 309-318.

Peeters, M. \& J. Rodríguez. 1999. Problemas bacterianos en la industria camaronera Ecuatoriana, prácticas de manejo y alternativas de control. El Mundo Acuícula 5: $13-18$.

Pipitone, C., F. Badalamenti, G.D'Anna \& B. Patti. 2000. Fish biomass increase after a four-year trawl ban in the Gulf of Castellammare (NW Sicily, Mediterranean Sea). Fish. Res. 48: 23-30.

Rengpipat, S., S. Rukpratanporn, S. Piyatiratitivorakul \& P. Menasaveta. 2000. Immunity enhancement in black tiger shrimp (Penaeus monodon) by a probiont bacterium (Bacillus S11). Aquaculture 191: 271-288.

Riquelme, C., G. Hayashida, A.E. Toranzo, J. Vilches \& P. Chavez. 1995. Pathogenicity studies on a Vibrio anguillarum-related (VAR) strain causing an epizootic in Argopecten purpuratus larvae cultured in Chile. Dis. Aquat. Org. 22: 135-141. 
Riquelme, C., R. Araya \& R. Escribano. 2000. Selective incorporation of bacteria by Argopecten purpuratus larvae: implications for the use of probiotics in culturing systems of the Chilean scallop. Aquaculture 181: 25-36.

Robertson, P.A.W., C.O'Dowd, C. Burrels, P. Williams \& B. Austin. 2000. Use of Carnobacterium sp. as a probiotic for Atlantic salmon (Salmo salar L.) and rainbow trout (Oncorhynchus mykiss, Walbaum). Aquaculture 185: 235-243.

Ruiz-Ponte, C., J.F. Samain, J.L. Sánchez \& J.L. Nicolas. 1999. The benefit of a Roseobacter species on the survival of scallop larvae. Mar. Biotechnol. 1: 52-59.

Sugumar, G., T. Nakai, Y. Hirata, D. Matsubara \& K. Muroga. 1998. Vibrio splendidus biovar II as the causative agent of bacillary necrosis of Japanese oyster Crassostrea gigas larvae. Dis. Aquat. Org. 33: $111-118$.

Torkildsen, L. \& T. Magnesen. 2004. Hatchery production of scallop larvae (Pecten maximus) survival in different rearing systems. Aquac. Int. 12: 489-507.
Torrento, M. \& J. Torres. 1996. In vitro inhibition of Vibrio harveyi by Pseudomonas sp. isolated from aquatic environment. UPV J. Nat. Sci. 1: 130-138.

Venkat, H.K., N.P. Shau \& K.J. Jain. 2004. Effect on feeding Lactobacillus-based probiotics on the gut microflora, growth and survival of postlarvae of Macrobrachium rosenbergii (de Man). Aquac. Res. 35: 501-507.

Villamil-Díaz, L. \& M.A. Martínez-Silva. 2009. Probióticos como herramienta biotecnológica en el cultivo de camarón: Reseña. Bol. Invest. Mar. Cost. 38: 165-187.

Vine, N.G., W.D. Leukes, H. Kaiser, J. Baxter \& T. Hecht. 2004. Competition for attachment of aquaculture candidate probiotic and pathogenic bacteria on fish intestinal mucus. J. Fish Dis. 27: 319-326.

Vine, N.G., W.D. Leukes \& K. Horst. 2006. Probiotics in marine larviculture. FEMS Microbiol. Rev. 30: 404-427.

Whitman, K.A. \& N.G. MacNair. 2004. Finfish and shellfish bacteriology manual. Techniques and procedures. Iowa state, Iowa, EEUU. 